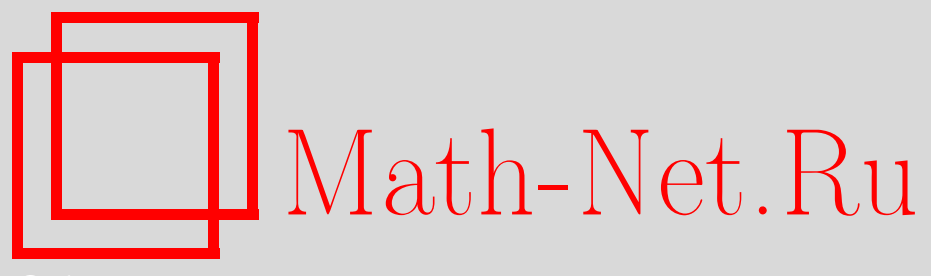

Ю. В. Быков, Решетки в одномерном кватернионном линейном пространстве, инвариантные относительно конечных линейных групп, Матем. заметки, 2011, том 89, выпуск 1, 122-126

DOI: https://doi.org/10.4213/mzm8923

Использование Общероссийского математического портала Math-Net.Ru подразумевает, что вы прочитали и согласны с пользовательским соглашением http://www . mathnet.ru/rus/agreement

Параметры загрузки:

IP: 3.85 .183 .62

26 апреля 2023 г., $17: 52: 48$

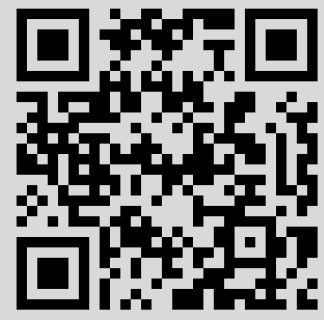




\section{Решетки в одномерном кватернионном линейном пространстве, инвариантные относительно конечных линейных групп}

\section{Ю. В. Быков}

Введение. Пусть $\mathbb{H}$ - тело кватернионов над $\mathbb{R}$, порожденное такими элементами $i$, $j, k$, что

$$
i^{2}=j^{2}=k^{2}=-1, \quad i j=-j i=k, \quad j k=-k j=i, \quad k i=-i k=j .
$$

Мы отождествляем $\mathbb{C}$ с подполем $\mathbb{R}+\mathbb{R} i$ в $\mathbb{H}$.

Пусть $V$ - правое $n$-мерное линейное пространство над $\mathbb{H}$. Элемент конечного порядка из $\mathrm{GL}(V)$ называется отражением, если пространство его неподвижных точек $(n-1)$-мерно. Все конечные подгруппы в $\mathrm{GL}(V)$, порожденные отражениями, классифицированы в [1]. Имея в виду перенесение на кватернионный случай методов полученной в [2] классификации дискретных групп, порожденных аффинными эрмитовыми отражениями, мы приходим к задаче об описании с точностью до подобия (т.е. умножения на ненулевой элемент из $\mathbb{H}$ ) решеток в $V$, инвариантных относительно таких конечных подгрупп в $\mathrm{GL}(V)$, которые порождены отражениями (под решеткой мы понимаем свободную подгруппу ранга $4 n$ в аддитивной группе $V$ ). В этой заметке такое описание дано при $n=1$. Оно существенно используется в исследовании общего случая, который будет рассмотрен отдельно.

Итак, далее мы считаем, что $V$ - это $\mathbb{H}$ с умножением на константы справа, $\operatorname{End}(V)-$ это $\mathbb{H}$, действующее на $V$ умножением слева, а $\mathrm{GL}(V)$ - мультипликативная группа $\mathbb{H}^{*}$ тела $\mathbb{H}$. Все неединичные элементы конечного порядка из $\mathbb{H}^{*}$ являются отражениями. Описание конечных подгрупп в $\mathbb{H}^{*}$ хорошо известно (см., например, [3]) и состоит в следующем (ниже через $\langle M\rangle$ обозначается подгруппа группы $G$, порожденная подмножеством $M \subseteq G):$

ПреДЛОЖЕНИЕ 1. С точностъю до сопряженности конечные подгруппы в $\mathbb{H}^{*}$ исчерпываются следующим списком попарно несопряженных подгрупп:

- $\mathbf{C}_{m}=\left\langle\varepsilon_{m}\right\rangle$, где $\varepsilon_{m}=e^{2 \pi i / m}-$ циклическая группа порядка $m$;

- $\mathbf{D}_{m}=\left\langle\mathbf{C}_{2 m}, k\right\rangle-$ бинарная диэдральная группа порядка $4 \mathrm{~m}$;

- $\mathbf{T}=\left\langle\mathbf{D}_{2}, \omega\right\rangle$, где $\omega=(-1+i+j+k) / 2$ - бинарная тетраэдралъная группа порядка 24 ;

- $\mathbf{O}=\langle\mathbf{T}, \alpha\rangle$, где $\alpha=(i-1) / \sqrt{2}$ - бинарная октаэдралъная группа порядка 48 ;

- $\mathbf{I}=\left\langle\mathbf{D}_{2}, \beta\right\rangle$, где $\beta=\cos (\pi / 5)+\cos (3 \pi / 5) i-j / 2$ - бинарная икосаэдральная группа порядка 120.

Далее обозначения из предложения 1 используются без напоминаний.

\section{Инвариантные решетки.}

ПреДложение 2. (i) $\mathbf{C}_{m}$-инвариантные решетки в $V$ существуют только при $m=$ $1,2,3,4 u 6$.

(i) При $m=1,2$ любая решетка в $V$ является $\mathbf{C}_{m}$-инвариантной.

(iii) Если $m=3,4,6$, то с точностъю до подобия множество $\mathbf{C}_{m}$-инвариантных решеток в $V$ совпадает с множеством всех подгрупп вида

$$
\left(\mathbb{Z}+\varepsilon_{m} \mathbb{Z}\right)+\left(\mathbb{Z}+\varepsilon_{m} \mathbb{Z}\right) \nu,
$$

где 1 и $\nu \in \mathbb{H}$ линейно независимы над $\mathbb{C}$ относительно умножения слева, а норма любого ненулевого элемента из подгруппы (1) не менъще 1. 
Доказательство. (i) Рассмотрим $V$ как 4-мерное линейное пространство над $\mathbb{R}$. В базисе $1, i, j, k$ матрица линейного оператора $\varepsilon_{m}$ имеет вид

$$
\left(\begin{array}{cc}
A & 0 \\
0 & A
\end{array}\right), \quad \text { где } \quad A=\left(\begin{array}{cc}
\cos \left(\frac{2 \pi}{m}\right) & -\sin \left(\frac{2 \pi}{m}\right) \\
\sin \left(\frac{2 \pi}{m}\right) & \cos \left(\frac{2 \pi}{m}\right)
\end{array}\right) .
$$

Значит, след этого оператора равен $4 \cos (2 \pi / m)$. Допустим, что в $V$ имеется $\mathbf{C}_{m}$-инвариантная решетка $\Gamma$. Любой ее базис является и базисом $V$ над $\mathbb{R}$. Ввиду $\mathbf{C}_{m}$-инвариантности Г матрица оператора $\varepsilon_{m}$ в таком базисе целочисленна. Поэтому след этого оператора является целым числом. Значит, если $\mathbf{C}_{m}$-инвариантная решетка в $V$ существует, то $4 \cos (2 \pi / m) \in \mathbb{Z}$. Отсюда легко следует утверждение (i).

(ii) Утверждение (ii) очевидно.

(iii) Ясно, что подгруппа (1) при любом $m$ является $\mathbf{C}_{m}$-инвариантной, и при $m=$ $3,4,6$ она является решеткой в $V$. Любая $\mathbf{C}_{m}$-инвариантная решетка $\Gamma$ в $V$ является конечно порожденным модулем без кручения над кольцом $\mathbb{Z}\left[\varepsilon_{m}\right]=\mathbb{Z}+\varepsilon_{m} \mathbb{Z}$ относительно умножения слева. Пусть $m=3,4,6$. Тогда это кольцо является кольцом главных идеалов $[4 ; \mathrm{I}, \S 4]$, а потому $\Gamma-$ свободный $\mathbb{Z}\left[\varepsilon_{m}\right]$-модуль. Поскольку $\operatorname{rk}_{\mathbb{Z}}(\Gamma)=4$,

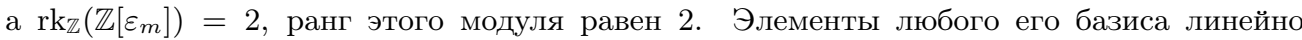
независимы над $\mathbb{C}$ относительно умножения слева, так как линейная оболочка $\Gamma$ над $\mathbb{R}$ (а потому и над $\mathbb{C}$ ) совпадает с $V$. Используя мультипликативность нормы и то, что норма любого ненулевого элемента из $\mathbb{Z}\left[\varepsilon_{m}\right]$ не меньше 1 , из известной теоремы о свободных модулях над кольцами главных идеалов $[5 ; \mathrm{XV}, \S 2$, теорема 5] мы выводим, что всякий ненулевой элемент $\gamma \in \Gamma$ минимальной нормы включается в базис $\mathbb{Z}\left[\varepsilon_{m}\right]$-модуля $Г$. Тогда $\Gamma \gamma^{-1}$ - решетка вида (1). Это доказывает утверждение (iii).

ПредЛОЖЕНИЕ 3. (i) Если в $V$ существует решетка, инвариантная относительно нециклической группы $\mathbf{D}_{m}$, то $m=2$ или 3.

(ii) $C$ точностью до подобия следующими двумя решетками исчерпываются все $\mathbf{D}_{2}$-инвариантные решетки в $V$ :

$$
\begin{gathered}
\mathbb{Z}+i \mathbb{Z}+j \mathbb{Z}+k \mathbb{Z}, \\
\mathbb{Z}+i \mathbb{Z}+j \mathbb{Z}+\left(\frac{1+i+j+k}{2}\right) \mathbb{Z} .
\end{gathered}
$$

(iii) C точностъю до подобия единственной $\mathbf{D}_{3}$-инвариантной решеткой в $V$ являemcя

$$
\left(\mathbb{Z}+\varepsilon_{6} \mathbb{Z}\right)+\left(\mathbb{Z}+\varepsilon_{6} \mathbb{Z}\right) k
$$

Доказательство. (i) $\mathbf{D}_{m}$-инвариантные решетки $\mathbf{C}_{2 m}$-инвариантны, так как $\mathbf{C}_{2 m} \subset$ $\mathbf{D}_{m}$. Отсюда и из предложения 2 вытекает утверждение (i) (так как $\mathbf{D}_{1}$ циклическая).

(ii) Из $\mathbf{D}_{2}=\left\langle\mathbf{C}_{4}, k\right\rangle$ и предложения 2 следует, что если $\mathbf{D}_{2}$-инвариантная решетка в $V$ существует, то она подобна решетке

$$
(\mathbb{Z}+i \mathbb{Z})+(\mathbb{Z}+i \mathbb{Z}) \nu
$$

где 1 и $\nu$ линейно независимы над $\mathbb{C}$ относительно умножения слева, а $\mathbf{D}_{2}$-инвариантность решетки (5) эквивалентна условию

$$
k((\mathbb{Z}+i \mathbb{Z})+(\mathbb{Z}+i \mathbb{Z}) \nu) \subseteq(\mathbb{Z}+i \mathbb{Z})+(\mathbb{Z}+i \mathbb{Z}) \nu .
$$

Выясним, при каком $\nu$ выполнено (6). Пусть $\nu=a+i b+j c+k d, a, b, c, d \in \mathbb{R}$. Заменяя $\nu$ на его сумму с подходящим элементом из $\mathbb{Z}+i \mathbb{Z}$, без ограничения общности можно считать, что

$$
|a| \leqslant \frac{1}{2}, \quad|b| \leqslant \frac{1}{2} .
$$


Условие (6) записывается в виде

$$
\begin{gathered}
(k \mathbb{Z}+j \mathbb{Z})+((a k+b j-c i-d) \mathbb{Z}+(a j-b k-c+d i) \mathbb{Z}) \\
\subseteq(\mathbb{Z}+i \mathbb{Z})+(\mathbb{Z}+i \mathbb{Z})(a+b i+c j+d k) .
\end{gathered}
$$

Поэтому оно эквивалентно тому, что для любых $(A, B, C, D) \in \mathbb{Z}^{4}$ следующая система уравнений относительно $X, Y, Z, W$

$$
\left\{\begin{array}{l}
A+C a-D b=-Z d-W c, \\
B+C b+D a=-Z c+W d, \\
C c-D d=Y+Z b+W a, \\
C d+D c=X+Z a-W b
\end{array}\right.
$$

имеет целочисленное решение. Предположим, что система (8) обладает этим свойством, и исследуем, какими могут быть $a, b, c, d$.

Положим $B=C=D=0, A=-1$. Из первого равенства системы (8) следует $c^{2}+d^{2} \neq 0$. Легко видеть, что в этом случае (8) имеет единственное решение

$$
\left\{\begin{array}{l}
X=\frac{-a d+b c}{c^{2}+d^{2}}, \\
Y=\frac{-b d-a c}{c^{2}+d^{2}}, \\
Z=\frac{d}{c^{2}+d^{2}}, \\
W=\frac{c}{c^{2}+d^{2}} .
\end{array}\right.
$$

Поскольку $|\nu|=\sqrt{a^{2}+b^{2}+c^{2}+d^{2}} \geqslant 1$ (см. предложение 2 (iii)), из (7) следует $c^{2}+d^{2} \geqslant$ $1 / 2$, откуда $0<Z^{2}+W^{2} \leqslant 2$, так как $Z^{2}+W^{2}=1 /\left(c^{2}+d^{2}\right)$. Значит, $Z^{2}+W^{2}=1$ или 2 , поскольку $Z$ и $W$ - целые числа.

Если $Z^{2}+W^{2}=1$, то $c^{2}+d^{2}=1$ и либо $Z= \pm 1, W=0$, либо $Z=0, W= \pm 1$. Из двух последних равенств (9) следует тогда, что либо $c=0, d= \pm 1$, либо $c= \pm 1, d=0$. Поскольку $X$ и $Y$ - целые числа, из первых двух равенств (9) следует, что и $a, b$ целые. Ввиду (7) получаем $a=b=0$. Значит, $\nu= \pm j$ или $\pm k$. Ясно, что при таком $\nu$ решетка (5) совпадает с решеткой (2). Отметим также, что, ввиду предложения 2 (iii), последнее показывает $\mathbf{C}_{4}$-инвариантность решетки (2), а значит, и $\mathbf{D}_{2}$-инвариантность, так как ее $k$-инвариантность очевидна.

Если же $Z^{2}+W^{2}=2$, то $c^{2}+d^{2}=1 / 2$ и $|Z|=|W|=1$. Из последних двух равенств (9) следует тогда, что $|c|=|d|=1 / 2$, а из первых двух, с учетом целочисленности $X$ и $Y$ и условий (7) и $|\nu| \geqslant 1$, следует, что $|a|=|b|=1 / 2$. Заменяя $\nu$ (в зависимости от знаков $c$ и $d$ ) на $\pm \nu$ или $\pm i \nu$, можно считать, не меняя решетки (5), что $c=d=1 / 2$. Заменяя затем элемент $\nu$ на его сумму с подходящим элементом из $\mathbb{Z}+i \mathbb{Z}$, можно считать, не меняя решетки (5), что $\nu=(1+i+j+k) / 2$. Легко видеть, что при таком $\nu$ решетка (5) совпадает с решеткой (3). Отметим также, что ввиду предложения 2 (iii) последнее показывает $\mathbf{C}_{4}$-инвариантность решетки (3). Поскольку, как нетрудно проверить, эта решетка $k$-инвариантна, это показывает, что она и $\mathbf{D}_{2}$-инвариантна. Это завершает доказательство утверждения (ii).

(iii) Из $\mathbf{D}_{3}=\left\langle\mathbf{C}_{6}, k\right\rangle$ и предложения 2 (iii) следует, что если $\mathbf{D}_{3}$-инвариантная решетка в $V$ существует, то она подобна решетке

$$
\left(\mathbb{Z}+\varepsilon_{6} \mathbb{Z}\right)+\left(\mathbb{Z}+\varepsilon_{6} \mathbb{Z}\right) \nu
$$

для которой

$$
k\left(\left(\mathbb{Z}+\varepsilon_{6} \mathbb{Z}\right)+\left(\mathbb{Z}+\varepsilon_{6} \mathbb{Z}\right) \nu\right) \subseteq\left(\mathbb{Z}+\varepsilon_{6} \mathbb{Z}\right)+\left(\mathbb{Z}+\varepsilon_{6} \mathbb{Z}\right) \nu
$$


или, эквивалентно,

$$
\left(\mathbb{Z}+\varepsilon_{6} \mathbb{Z}\right)+\left(\mathbb{Z}+\varepsilon_{6} \mathbb{Z}\right) \nu \subseteq k\left(\left(\mathbb{Z}+\varepsilon_{6} \mathbb{Z}\right)+\left(\mathbb{Z}+\varepsilon_{6} \mathbb{Z}\right) \nu\right)
$$

Выясним, при каком $\nu$ выполнено (11). Пусть $\nu=a+i b+j c+k d, a, b, c, d \in \mathbb{R}$. Поскольку $\varepsilon_{6}=(1+i \sqrt{3}) / 2$, заменяя элемент $\nu$ на его сумму с подходящим элементом из $\mathbb{Z}+\varepsilon_{6} \mathbb{Z}$, без ограничения общности можно считать, что выполнено условие

$$
|a| \leqslant \frac{1}{2}, \quad|b| \leqslant \frac{\sqrt{3}}{4} .
$$

Условие (11) записывается в виде условия

$$
\begin{aligned}
& k \mathbb{Z}+\left(\frac{j \sqrt{3}+k}{2}\right) \mathbb{Z}+(-d-i c+j b+k a) \mathbb{Z}+\left(\frac{k a+j b-i c-d}{2}+(j a-k b-c+i d) \frac{\sqrt{3}}{2}\right) \mathbb{Z} \\
& \supseteq \mathbb{Z}+\left(\frac{1+i \sqrt{3}}{2}\right) \mathbb{Z}+(a+i b+j c+k d) \mathbb{Z} \\
& \quad+\left(\frac{a+i b+j c+k d}{2}+(-b+i a+c k-j d) \frac{\sqrt{3}}{2}\right) \mathbb{Z}
\end{aligned}
$$

которое, в свою очередь, эквивалентно тому, что для любых $(A, B, C, D) \in \mathbb{Z}^{4}$ следующая система уравнений относительно $X, Y, Z, W$

$$
\left\{\begin{array}{l}
-C d-\frac{D(d+\sqrt{3} c)}{2}=X+\frac{Y}{2}+Z a+\frac{W(a-\sqrt{3} b)}{2}, \\
-C c-\frac{D(c-\sqrt{3} d)}{2}=\frac{\sqrt{3} Y}{2}+Z b+\frac{W(b+\sqrt{3} a)}{2}, \\
\frac{\sqrt{3} B}{2}+C b+\frac{D(b+\sqrt{3} a)}{2}=Z c+\frac{W(c-\sqrt{3} d)}{2}, \\
A+\frac{B}{2}+C a+\frac{D(a-\sqrt{3} b)}{2}=Z d+\frac{W(d+\sqrt{3} c)}{2}
\end{array}\right.
$$

имеет целочисленное решение. Предположим, что эта система обладает указанным свойством, и исследуем, какими могут быть $a, b, c, d$. Заметим, что из $|\nu| \geqslant 1$ и (12) следует неравенство

$$
c^{2}+d^{2} \geqslant \frac{9}{16} \text {. }
$$

Положим далее $A=1, B=C=D=0$. Тогда из последних двух уравнений системы (13) мы получаем, что

$$
\left\{\begin{array}{l}
c=\frac{\sqrt{3} W}{2\left(Z^{2}+Z W+W^{2}\right)}, \\
d=\frac{2 Z+W}{2\left(Z^{2}+Z W+W^{2}\right)},
\end{array}\right.
$$

откуда следует равенство $c^{2}+d^{2}=1 /\left(Z^{2}+Z W+W^{2}\right)$. Поскольку $Z$ и $W-$ целые числа, из этого равенства и (14) легко вывести, что $(Z, W)=( \pm 1,0),(0, \pm 1)$ или $( \pm 1, \mp 1)$. Рассмотрим каждую из этих возможностей отдельно.

Пусть $(Z, W)=(0,1)$. Тогда из $(15)$ следует, что $c=\sqrt{3} / 2, d=1 / 2$, а из первых двух уравнений системы (13) - что $a=-Y-X / 2, b=(4 Y+X) \sqrt{3} / 2$. Поскольку $X$ и $Y-$ целые числа, отсюда и из (12) следует, что $a=b=0$. Итак, в этом случае $\nu=(j \sqrt{3}+k) / 2=\varepsilon_{6}^{5} k$.

В остальных случаях аналогичное рассуждение дает следующее.

Если $(Z, W)=(0,-1)$, то $c=-\sqrt{3} / 2, d=-1 / 2, a=Y+X / 2, b=-X \sqrt{3} / 2$, откуда $a=b=0$ и, значит, $\nu=-(j \sqrt{3}+k) / 2=\varepsilon_{6}^{2} k$. 
Если $(Z, W)=(1,0)$, то $c=0, d=1, a=-X-Y / 2, b=-Y \sqrt{3} / 2$, откуда $a=b=0$ и, значит, $\nu=k$.

Если $(Z, W)=(-1,0)$, то $c=0, d=-1, a=X+Y / 2, b=Y \sqrt{3} / 2$, откуда $a=b=0$ и, значит, $\nu=-k$.

Если $(Z, W)=(1,-1)$, то $c=-\sqrt{3} / 2, d=1 / 2, a=(-X+Y) / 2, b=(-3 X+Y) \sqrt{3} / 2$, откуда $a=b=0$ и, значит, $\nu=(-j \sqrt{3}+k) / 2=\varepsilon_{6} k$.

Если $(Z, W)=(-1,1)$, то $c=\sqrt{3} / 2, d=-1 / 2, a=(X-Y) / 2, b=(X+Y) \sqrt{3} / 2$, откуда $a=b=0$ и, значит, $\nu=(j \sqrt{3}-k) / 2=\varepsilon_{6}^{4} k$.

Ясно, что в каждом из этих случаев при найденном значении $\nu$ решетка (10) совпадает с решеткой (4).

Чтобы завершить доказательство утверждения (iii), остается заметить, что решетка (4) является $\mathbf{C}_{6}$-инвариантной в силу предложения 2 (iii) и $k$-инвариантной ввиду равенства $k \varepsilon_{6}=\varepsilon_{6}^{5} k$. Значит, она $\mathbf{D}_{3}$-инвариантна.

ЗАмечАние 1 . Легко проверить, что решетка (2) не инвариантна относительно $\omega$ и $\beta$, a (3) инвариантна относительно $\omega$ и не инвариантна относительно $\alpha$ и $\beta$.

ПреДЛОЖенИЕ 4. (i) C точностъю до подобия единственной Т-инвариантной решеткой в $V$ является решетка (3).

(ii) $B V$ не существует $\mathbf{O}$-инвариантных решеток.

(iii) $B V$ не существует I-инвариантных решеток.

ДокАзАтЕЛЬСтво. Утверждения (i) и (iii) следуют из предложения 3, замечания 1 и, соответственно, равенств $\mathbf{T}=\left\langle\mathbf{D}_{2}, \omega\right\rangle$ и $\mathbf{I}=\left\langle\mathbf{D}_{2}, \beta\right\rangle$, а утверждение (ii) - из утверждения (i), замечания 1 и равенства $\mathbf{O}=\langle\mathbf{T}, \alpha\rangle$.

\section{СПИСОК ЦИТИРОВАННОЙ ЛИТЕРАТУРЫ}

[1] A. M. Cohen, J. Algebra, 64:2 (1980), 293-324. [2] V. L. Popov, Discrete Complex Reflection Groups, Commun. Math. Inst. Rijksuniv. Utrecht, 15, Rijksuniv. Utrecht, Utrecht, 1982. [3] P. Du Val, Homographies, Quaternions and Rotations, Oxford Math. Monogr., Clarendon Press, Oxford, 1964. [4] К. Айерлэнд, М. Роузен, Классическое введение в современную теорию чисел, Мир, М., 1987. [5] С. Ленг, Алгебра, Мир, М., 1968.

\section{Ю. В. Быков}

Московский государственный институт электроники и математики (технический университет)

E-mail: yurikbykov@mail.ru
Поступило

26.03.2010 